\title{
Correlation in Repeated Games with Public
}

\section{Monitoring}

Pavlo Prokopovych (Kyiv School of Economics and Kyiv Economics Institute) 


\title{
Correlation in Repeated Games with Public Monitoring*
}

\author{
Pavlo Prokopovych ${ }^{\dagger}$ \\ KSE and KEI, Kyiv, Ukraine
}

January 24, 2008

\footnotetext{
${ }^{*}$ An earlier version of this paper is part of Chapter 3 of my doctoral thesis (2005) at Michigan.

$\dagger$ Email: prokop@eerc.kiev.ua, Phone: 38-044-492-8012, Fax: 38-044-492-8011, Addresses: Kyiv School of Economics, 51 Dehtyarivska, 2nd floor, suite 12, Kyiv 03113, Ukraine; Kyiv Economics Institute, 41 Nyzhniy Val St., Office 55, Kyiv 04070, Ukraine
} 


\begin{abstract}
This paper studies correlation in discounted infinitely repeated games with public monitoring. Two extensions of the perfect public equilibrium are proposed: the public correlated equilibrium, where a correlation device sends private correlated messages to the players at the beginning of each period, and the public augmented equilibrium, where the device also publicly informs the players of the recommended action profile at the end of each period. The set of public correlated equilibrium payoffs is compared to the set of subgame perfect publicly correlated equilibrium payoffs in the perfect monitoring case. It is shown that augmented correlation produces efficiency gains in the repeated partnership game by Radner et al. (1986).
\end{abstract}

JEL classification: $\mathrm{C} 72 ; \mathrm{C} 73$

Keywords: Repeated Games; Extensive form correlation; Efficiency; Imperfect public monitoring; Perfect monitoring 


\section{Introduction}

This paper studies discounted infinitely repeated $n$-player games with public monitoring, endowed with an extensive form correlation device. This structure captures situations of repeated interaction among players who choose their actions conditional on both public and correlated private information.

A general framework for the analysis of repeated games with imperfect public monitoring was provided by Abreu et al. (1990) (APS). Building on their work, Fudenberg et al. (1994) have shown that imperfect monitoring imposes essentially no restriction on the set of equilibrium payoffs if the signal space is large enough. Otherwise, the equilibrium payoff set may be bounded away from the Pareto frontier even as the discount factor tends to one (Radner et al. 1986; Fudenberg at al. 1994, pp. 1003-04). This inefficiency disappears if communication among players is allowed and the number of players is at least four (Kandori 2003). Sometimes efficiency can be improved by employing private strategies which depend not only on the history of public signals but also on players' own actions in the past (Kandori and Obara 2006a). Another approach to dealing with the inefficency problem is based on assuming reduced sensitivity of the public signal to the hidden actions of the players (Kandori and Obara 2006b).

The focus of this paper is also on infinitely repeated games with imperfect public monitoring. Following Forges (1986) and Lehrer (1992), we add an extensive form correlation device to the infinitely repeated game. The device selects a vector of messages, one for each player, in every period of the game, based on the history of public signals. In our setting, the device is not a communication device since it does not receive any inputs from players (Myerson 1986; Tomala 2005). Players

condition their play in every period on $(i)$ the history of public signals and $(i i)$ the 
latest private message of the device. The distribution according to which the device randomly selects its recommendations in every period depends only on the history of realizations of the public signal. As a result, each non-empty public history may be treated as if it gives rise to a proper subgame. In repeated games with perfect monitoring, a related solution concept is that of subgame perfect publicly correlated equilibrium by Myerson (1991, p. 334), where, in every period, the correlation device publicly recommends an action profile to the players.

In the APS spirit, we decompose an equilibrium payoff vector into an admissible pair consisting of a probability distribution on the product of the players' action sets and a continuation value function. This extension leads to a number of useful characterizations of the set of public correlated equilibrium payoffs.

We then introduce augmented correlation devices. In every period, these devices first send players private recommendations and, after the players have moved, publicly inform them of the whole recommended action profile. Obviously, in the perfect monitoring case, the corresponding equilibrium payoff set contains both the set of public correlated equilibrium payoffs and the set of subgame perfect public randomization (publicly correlated) equilibrium payoffs. However, the opportunities opened up by augmented correlation are not confined to introducing public randomization effects into the model. Augmentation enriches public histories with previously recommended action profiles and makes it easier to identify deviators.

A similar enrichment of public histories results from asking the players to publicly announce which actions they have just taken at the end of each period. However, ensuring players' truth telling leads to additional conditions on the number of players (Kandori 2003). In its turn, augmentation is not about the action profile just chosen by the players: the mediator publicly announce its own private recommendations to the players at the end of each period. As we will show below by example, such 
"leaks" of players' private information may lead to improved efficiency in repeated games with imperfect public monitoring.

In Example 1, we study an infinitely repeated prisoners' dilemma game with perfect monitoring. For a fixed discount factor, we show that the corresponding set of subgame perfect public randomization equilibrium payoffs is a proper subset of the set of public correlated equilibrium payoffs. That is, imperfect correlation may produce more efficient outcomes than perfect correlation. The intution behind this is that of Aumann (1974)'s, but applied to the infinitely repeated game. At the same time, it is worth noting that the stage game of the repeated game has a unique Nash equilibrium. In general, the set of subgame perfect public randomization equilibrium payoffs may strictly contain the set of public correlated equilibrium payoffs since the history of past public messages is common knowledge in the presence of a public randomization device.

As we show for an infinitely repeated prisoners' dilemma game (Example 2), extensive form correlation alone may produce no efficiency improvement if public monitoring is imperfect and the signal space is small. However, by publicly announcing the most recent private recommendations at the end of each period, the mediator can reduce the welfare loss caused by the imperfect observability of actions.

\section{Theoretical Underpinnings}

In the stage game $G_{Y}$, players move simultaneously, and player $i \in N=\{1, \ldots, n\}$ chooses an action $a_{i}$ from a finite set $A_{i}$. Every profile of actions $a \in A=\underset{i \in N}{\times} A_{i}$ induces a probability distribution on $Y=\left\{y_{1}, \ldots, y_{r}\right\}$, the finite set of publicly observable signals. Let $p(y \mid a)$ denote the probability of $y \in Y$ given $a \in A$. The stage game payoff function for player $i$ is given by $\pi_{i}\left(a_{i}, y\right)$. Player $i$ 's expected stage game 
payoff from action profile $a$ is

$$
g_{i}(a)=\sum_{y \in Y} p(y \mid a) \pi_{i}\left(a_{i}, y\right)
$$

The repeated game $G_{Y}^{\infty}(\delta)$ begins at time 0 , with the null history $h^{0}=\{\varnothing\}$. Let $H_{Y}^{k}$ denote the set of all possible period- $k$ public histories $h_{Y}^{k}=\left\{y^{0}, \ldots, y^{k-1}\right\}$. Players discount future payoffs with a common discount factor $\delta \in(0,1)$, and player $i$ 's objective is to maximize

$$
(1-\delta) \sum_{k=0}^{\infty} \delta^{k} g_{i}\left(a^{k}\right)
$$

where $a^{k}$ refers to the action profile chosen in period $k$. Adding the subscript " $Y$ " to $G$ emphasizes that actions are imperfectly observed. However, the formulation also encompasses the case of perfect observability of actions. So, with a slight abuse of notation, we will denote by $g_{i}$ player $i$ 's payoff function in repeated games with perfectly observable actions as well.

\subsection{Correlation Devices}

An extensive form correlation device $C$ sends separately and confidentially messages to the players at the beginning of each period. Formally, $C=\left(\left(M_{i}\right)_{i \in N}, \mu\right)$ is an $(n+1)$-tuple consisting of a family of finite message sets $M_{i}$ and a map $\mu$ acts from the set $H_{Y}=\bigcup_{k=0}^{\infty} H_{Y}^{k}$ of public histories to the set $\triangle(M)$ of probability distributions on $M=\underset{i \in N}{\times} M_{i}$. The map $\mu$ is common knowledge among the players. Let $\mu(m=$ $\left.\left(m_{1}, \ldots, m_{n}\right) \mid h_{Y}\right)$ denote the conditional probability that the correlation device will send each player $i$ message $m_{i}$ at $h_{Y}$. After public history $h_{Y}$, the device selects $m=\left(m_{1}, \ldots, m_{n}\right) \in M$ according to $\mu\left(\cdot \mid h_{Y}\right)$ and privately informs each player $i$ of 
$m_{i}$. A strategy for player $i$ is a map $f_{i}$ from $H_{Y} \times M_{i}$ to $\triangle\left(A_{i}\right)$. Let $f_{i}\left(a_{i} \mid h_{Y}, m_{i}\right)$ represent the probability that player $i$ will choose $a_{i}$ conditional on $h_{Y}$ and $m_{i}$. For a correlation device $C$ and a strategy profile $f=\left(f_{1}, \ldots, f_{n}\right)$, we can compute the following:

the probability $\mu_{C, f}\left(a \mid h_{Y}\right)=\sum_{m \in M^{i}} \prod_{i=1}^{n} f_{i}\left(a_{i} \mid h_{Y}, m_{i}\right) \mu\left(m \mid h_{Y}\right)$ that $a$ will be played at $h_{Y}$;

the probability $p_{C, f}\left(y \mid h_{Y}\right)=\sum_{a \in A} p(y \mid a) \mu_{C, f}\left(a \mid h_{Y}\right)$ that $y$ will occur at $h_{Y}$;

the probability $p_{C, f}\left(h_{Y}^{k+1}\right)$ that $h_{Y}^{k+1}=\left\{y^{0}, \ldots, y^{k}\right\}$ will take place in the first $k$ periods

$$
p_{C, f}\left(h_{Y}^{k+1}\right)=p_{C, f}\left(y^{0} \mid h^{0}\right) p_{C, f}\left(y^{1} \mid y^{0}\right) \ldots p_{C, f}\left(y^{k} \mid\left\{y^{0}, \ldots y^{k-1}\right\}\right)
$$

the probability that $\left(a^{k}, y^{k}\right)$ will occur in period $k$

$$
p_{C, f}^{k}\left(a^{k}, y^{k}\right)=\sum_{h_{Y}^{k} \in H_{Y}^{k}} p\left(y^{k} \mid a^{k}\right) \mu_{C, f}\left(a^{k} \mid h_{Y}^{k}\right) p_{C, f}\left(h_{Y}^{k}\right)
$$

and player $i$ 's expected average discounted payoff

$$
u_{i}(f \mid \delta, C)=(1-\delta) \sum_{k=0}^{\infty} \delta^{k} \sum_{\left(a^{k}, y^{k}\right) \in A \times Y} p_{C, f}^{k}\left(a^{k}, y^{k}\right) \pi_{i}\left(a_{i}^{k}, y^{k}\right)
$$

Having the players' set of strategies and payoff functions defined, we denote this extended repeated game by $G_{C}^{\infty}(\delta)$. Then an extensive form correlated equilibrium of $G_{Y}^{\infty}(\delta)$ extended by $C$ can be defined as a Nash equilibrium of $G_{C}^{\infty}(\delta)$.

Definition 1 An extensive form correlated equilibrium $(C, f)$ of $G_{Y}^{\infty}(\delta)$ is a pair consisting of an extensive form correlation device $C$ and a strategy profile $f$ such that the strategy profile is a Nash equilibrium of the extended game induced by the device, 
that is, $u_{i}(f \mid \delta, C) \geq u_{i}\left(f_{i}^{\prime}, f_{-i} \mid \delta, C\right)$ for all $f_{i}^{\prime}: H_{Y} \times M_{i} \mapsto \triangle\left(A_{i}\right)$ and all $i \in N$.

Further on, we will focus on public correlated equilibria that for each period $k$ and public history $h_{Y}^{k}$ yield a Nash equilibrium of the corresponding extended game from that period on.

For any correlation device $C$, strategy profile $f$, and history $h_{Y}$, we denote by $C^{\mid h_{Y}}=\left(\left(M_{i}\right)_{i \in N}, \mu^{\mid h_{Y}}\right)$ the correlation device induced by $C$ following $h_{Y}$, where $\mu^{\mid h_{Y}}$ is the map from $H_{Y}$ to $M=\underset{i \in N}{\times} M_{i}$ such that $\mu^{\mid h_{Y}}\left(m \mid h_{Y}^{\prime}\right)=\mu\left(m \mid\left\{h_{Y}, h_{Y}^{\prime}\right\}\right)$ for all $m \in M$ and $h_{Y}^{\prime} \in H_{Y}$; by $f^{\mid h_{Y}}=\left(f_{1}^{\mid h_{Y}}, \ldots, f_{n}^{\mid h_{Y}}\right)$ the strategy profile induced by $f$ following $h_{Y}$, where $f_{i}^{\mid h_{Y}}\left(a_{i} \mid h_{Y}^{\prime}, m_{i}\right)=f_{i}\left(a_{i} \mid\left\{h_{Y}, h_{Y}^{\prime}\right\}, m_{i}\right)$ for all $i \in N$, $m_{i} \in M_{i}, a_{i} \in A_{i}$, and $h_{Y}^{\prime} \in H_{Y}$; by $\mu^{0 \mid h_{Y}}=\mu^{\mid h_{Y}}\left(\cdot \mid h^{0}\right)$ the probability distribution on $M$ according to which the extensive form device $C=\left(\left(M_{i}\right)_{i \in N}, \mu\right)$ selects its recommendations at $h_{Y}$; by $f_{i}^{0 \mid h_{Y}}$ player $i$ 's strategy $f_{i}^{\mid h_{Y}}\left(\cdot \mid h^{0}\right): M_{i} \mapsto \triangle\left(A_{i}\right)$ at $h_{Y}$. Note that $C=C^{\mid h^{0}}$ and $f=f^{\mid h^{0}}$.

Definition 2 An extensive form correlated equilibrium $(C, f)$ of $G_{Y}^{\infty}(\delta)$ is called public correlated if $\left(C^{\mid h_{Y}}, f^{\mid h_{Y}}\right)$ is an extensive form correlated equilibrium of $G_{Y}^{\infty}(\delta)$ for any $h_{Y} \in H_{Y}$.

Given a strategy profile $f$ and a correlation device $C$, player $i$ 's expected average discounted payoff at $h_{Y} \in H_{Y}$ can be represented as

$u_{i}\left(f^{\mid h_{Y}} \mid \delta, C^{\mid h_{Y}}\right)=\sum_{(a, y) \in A \times Y} p(y \mid a) \mu_{C, f}\left(a \mid h_{Y}\right)\left((1-\delta) \pi_{i}\left(a_{i}, y\right)+\delta u_{i}\left(f^{\mid\left\{h_{Y}, y\right\}} \mid \delta, C^{\mid\left\{h_{Y}, y\right\}}\right)\right)$,

The following statement is the one-shot deviation principle for infinitely repeated games with public monitoring and extensive form correlation.

Proposition 1 A pair $(C, f)$ consisting of an extensive form correlation device $\left(\left(M_{i}\right)_{i \in N}, \mu\right)$ and a strategy profile $f=\left(f_{1}, \ldots, f_{n}\right), f_{i}: H_{Y} \times M_{i} \mapsto \triangle\left(A_{i}\right)$, is a 
public correlated equilibrium of $G_{Y}^{\infty}(\delta)$ if and only if, for any history $h_{Y} \in H_{Y}$, the correlation device $C^{0 \mid h_{Y}}=\left(\left(M_{i}\right)_{i \in N}, \mu^{0 \mid h_{Y}}\right)$, and the strategy profile $f^{0 \mid h_{Y}}=\left(f_{1}^{0 \mid h_{Y}}, \ldots\right.$, $\left.f_{n}^{0 \mid h_{Y}}\right), f_{i}^{0 \mid h_{Y}}: M_{i} \mapsto \triangle\left(A_{i}\right)$, form a correlated equilibrium of the game

$$
G_{Y}^{0 \mid h_{Y}}(\delta)=\left(N,\left(A_{i}\right)_{i \in N},\left(\sum_{y \in Y} p(y \mid a)\left((1-\delta) \pi_{i}\left(a_{i}, y\right)+\delta u_{i}\left(f^{\mid\left\{h_{Y}, y\right\}} \mid \delta, C^{\mid\left\{h_{Y}, y\right\}}\right)\right)\right)_{i \in N}\right) .
$$

Because of discounting, the proof of Proposition 1 reduces to a conventional backward induction argument.

It is useful to note that the revelation principle holds in this setting. An extensive form correlation device $C=\left(\left(M_{i}\right)_{i \in N}, \mu\right)$ is direct if $M_{i}=A_{i}$ for all $i \in N$. The obedient strategy for player $i$ is a map $f_{i}$ from $H_{Y} \times A_{i}$ to $A_{i}$ such that $f_{i}\left(a_{i} \mid h_{Y}, a_{i}\right)=$ 1 for all $h_{Y} \in H_{Y}$ and $a_{i} \in A_{i}$. An extensive form direct correlated equilibrium is just a map $\mu$ from $H_{Y}$ to $\triangle(A)$ such that the obedient strategy profile is a Nash equilibrium of the induced extended game. An extensive form direct correlated equilibrium $\mu$ of $G^{\infty}(\delta)$ is called public correlated if $\mu^{h_{Y}}$ is an extensive form direct correlated equilibrium of $G_{Y}^{\infty}(\delta)$ for any $h_{Y} \in H_{Y}$.

Corollary 1 Every public correlated equilibrium payoff vector of $G_{Y}^{\infty}(\delta)$ can be attained in a public direct correlated equilibrium of $G_{Y}^{\infty}(\delta)$.

It follows from the revelation principle for normal form games and Proposition 1. So further on, we assume that every extensive form correlation device added to $G_{Y}^{\infty}(\delta)$ is a direct device.

Now we show that the APS technique can be easily extended to repeated games with this kind of extensive form correlation. Let $V$ denote the set of feasible and individually weakly rational payoffs of $G_{Y}=\left(N,\left(A_{i}\right)_{i \in N},\left(g_{i}\right)_{i \in N}\right)$ and $V_{C}(\delta)$ the set of public correlated equilibrium payoffs of $G_{Y}^{\infty}(\delta)$. It is not difficult to see that every 
perfect public equilibrium strategy profile of $G_{Y}^{\infty}(\delta)$ can be represented as a public direct correlated equilibrium of $G_{Y}^{\infty}(\delta)$.

Let $M(\delta)$ denote the set of public correlated equilibrium strategy profiles of $G_{Y}^{\infty}(\delta)$ and $u_{i}\left(\mu^{\mid h_{Y}}, \delta\right)$ player $i$ 's expected average discounted payoff in a public direct correlated equilibrium $\mu$ at $h_{Y}$. If $\mu \in M(\delta)$, then $u\left(\mu^{\mid h_{Y}} \mid \delta\right) \in V_{C}(\delta)$ for any $h_{Y} \in H_{Y}$, and

$u_{i}\left(\mu^{\mid h_{Y}} \mid \delta\right)=\sum_{(a, y) \in A \times Y} p(y \mid a) \mu\left(a \mid h_{Y}\right)\left((1-\delta) \pi_{i}\left(a_{i}, y\right)+\delta u_{i}\left(\mu^{\mid\left\{h_{Y}, y\right\}} \mid \delta\right)\right), i=1, \ldots, n$.

By definition,

$$
V_{C}(\delta)=\left\{v \in \mathbb{R}^{n}: v=\left(u_{1}(\mu, \delta), \ldots, u_{n}(\mu, \delta)\right) \text { for some } \mu \in M(\delta)\right\}
$$

That is, if $v \in V_{C}(\delta)$, there exist a probability distribution $\mu \in \triangle(A)$, a function $\kappa$ from $Y$ to $V_{C}(\delta)$ such that $v_{i}=\sum_{(a, y) \in A \times Y} p(y \mid a) \mu(a)\left((1-\delta) \pi_{i}\left(a_{i}, y\right)+\delta \kappa_{i}(y)\right), i=$ $1, \ldots, n$, and no player has incentive to disobey any possible recommendation by the device. This observation is key to obtaining a number of powerful characterizations of $V_{C}(\delta)$.

Definition 3 Given $\delta \in(0,1)$, for any $W \subset \mathbb{R}^{n}$, a pair $(\mu, \kappa)$, where $\mu$ is a probability distribution on $A, \kappa$ is a function from $Y$ to $W$, is called $C$-admissible with respect to $W$ if, for all $a_{i} \in A_{i}, a_{i}^{\prime} \in A_{i}-\left\{a_{i}\right\}$, and $i \in N$,

$$
\begin{aligned}
\sum_{a_{-i} \in A_{-i} y \in Y} \sum_{i} p\left(y \mid a_{i}, a_{-i}\right) \mu\left(a_{i}, a_{-i}\right)\left((1-\delta) \pi_{i}\left(a_{i}, y\right)+\delta \kappa_{i}(y)\right) & \\
& \geq \sum_{a_{-i} \in A_{-i} y \in Y} \sum_{i} p\left(y \mid a_{i}^{\prime}, a_{-i}\right) \mu\left(a_{i}, a_{-i}\right)\left((1-\delta) \pi_{i}\left(a_{i}^{\prime}, y\right)+\delta \kappa_{i}(y)\right) .
\end{aligned}
$$


For $W \subset \mathbb{R}^{n}$, we define

$$
\begin{gathered}
B_{C}(W \mid \delta)=\left\{v \in \mathbb{R}^{n}: v_{i}=\sum_{(a, y) \in A \times Y} p(y \mid a) \mu(a)\left((1-\delta) \pi_{i}\left(a_{i}, y\right)+\delta \kappa_{i}(y)\right), i=1, \ldots, n,\right. \\
\quad \text { for some pair }(\mu, \kappa) C \text {-admissible w.r.t. } W\} .
\end{gathered}
$$

It is obvious that $V_{C}(\delta) \subset B_{C}\left(V_{C}(\delta) \mid \delta\right)$. Following APS, $W \subset \mathbb{R}^{n}$ is self-generating if $W \subset B_{C}(W \mid \delta)$. If $W \subset \mathbb{R}^{n}$ is bounded and self-generating, then $B_{C}(W \mid \delta) \subset$ $V_{C}(\delta)$. It is not difficult to show that $V_{C}(\delta)=B_{C}\left(V_{C}(\delta) \mid \delta\right)$ and $V_{C}(\delta)$ is a compact set. The proofs of the just mentioned properties are similar to those provided by APS for repeated games with imperfect public monitoring.

\subsection{Augmented Correlation Devices}

We now introduce devices that send players correlated private messages at the beginning of each stage and, after they have moved, publicly inform all of them of the whole recommended action profile. As a result, public histories also include past public messages. As we show below, the opportunities provided by such devices are not confined to correlation and public randomization.

Let $d^{k}=\left(d_{1}^{k}, \ldots, d_{n}^{k}\right)$ denote the action profile recommended by the device in period $k$ and $H_{D}^{k}$ the set of all possible period- $k$ public histories $h_{D}^{k}=\left(d^{0}, \ldots, d^{k-1}, y^{0}, \ldots, y^{k-1}\right)$.

As before, an extensive form augmented direct correlation device $D$ can be described

as a map $\mu_{D}$ from $H_{D}=\bigcup_{k=0}^{\infty} H_{D}^{k}$ to $\triangle(A)$. It sends messages to players twice every period. First, it separately and confidentially recommends an action to each player and then, after the players have moved, it publicly announces the recommended action profile. Now the device's recommendations may stochastically depend not only on previously observed public signals but also on its own past recommendations. 
A strategy for player $i$ is a map $f_{i}$ from $H_{D} \times A_{i}$ to $\triangle\left(A_{i}\right)$. Let $f_{i}\left(a_{i} \mid h_{D}, d_{i}\right)$ denote the probability that player $i$ will choose action $a_{i}$ according to $f_{i}$ conditional on $h_{D} \in H_{D}$ and $d_{i} \in A_{i}$. For an extensive form augmented correlation device $D$ and a strategy profile $f=\left(f_{1}, \ldots, f_{n}\right)$, one can compute player $i$ 's expected average discounted payoff $u_{i}(f \mid \delta, D)$.

Definition 4 An extensive form augmented correlated equilibrium $(D, f)$ of $G_{Y}^{\infty}(\delta)$ is a pair consisting of an extensive form augmented direct correlation device $D$ and an obedient strategy profile $f$ such that the strategy profile is a Nash equilibrium of the extended game induced by the device, that is,

$$
u_{i}(f \mid \delta, D) \geq u_{i}\left(f_{i}^{\prime}, f_{-i} \mid \delta, D\right) \text { for all } f_{i}^{\prime}: H_{D} \times A_{i} \mapsto \triangle\left(A_{i}\right) \text { and all } i \in N
$$

Definition 5 An extensive form augmented correlated equilibrium $(D, f)$ of $G_{Y}^{\infty}(\delta)$ is called public augmented if $\left(D^{\mid h_{D}}, f^{\mid h_{D}}\right)$ is an extensive form augmented correlated equilibrium of $G_{Y}^{\infty}(\delta)$ for any history $h_{D} \in H_{D}$.

The following statement describes the recursive structure of a public augmented equilibrium of $G_{Y}^{\infty}(\delta)$.

Proposition 2 A pair $(D, f)$, consisting of an extensive form augmented correlation device $\left(\left(A_{i}\right)_{i \in N}, \mu_{D}\right)$ and an obedient strategy profile $f=\left(f_{1}, \ldots, f_{n}\right), f_{i}: H_{D} \times$ $A_{i} \mapsto A_{i}$, is a public augmented equilibrium of $G_{Y}^{\infty}(\delta)$ if and only if, after any history $h_{D} \in H_{D}$,

$$
\begin{aligned}
& \sum_{d_{-i} \in A_{-i}} \sum_{y \in Y} p(y \mid d) \mu_{D}\left(d \mid h_{D}\right)\left((1-\delta) \pi_{i}\left(d_{i}, y\right)+\delta u_{i}\left(f^{\mid\left\{h_{D},(d, y)\right\}} \mid \delta, D^{\mid\left\{h_{D},(d, y)\right\}}\right)\right. \\
\geq & \sum_{d_{-i} \in A_{-i}} \sum_{y \in Y} p\left(y \mid a_{i}, d_{-i}\right) \mu_{D}\left(d \mid h_{D}\right)\left((1-\delta) \pi_{i}\left(a_{i}, y\right)+\delta u_{i}\left(f^{\mid\left\{h_{D},(d, y)\right\}} \mid \delta, D^{\mid\left\{h_{D},(d, y)\right\}}\right)\right.
\end{aligned}
$$


for all $d_{i} \in A_{i}, a_{i} \in A_{i}-\left\{d_{i}\right\}$, and $i \in N$.

We denote by $V_{D}(\delta)$ the set of public augmented equilibrium payoffs of $G_{Y}^{\infty}(\delta)$. The definitions of an admissible pair and the self-generating operator for the case of augmented correlation are straightforward. Let, for $a_{i} \in A_{i}, d \in A, i \in N$, and $\kappa: A \times Y \mapsto \mathbb{R}^{n}$,

$$
h_{i}\left(a_{i}, d, \kappa_{i}\right)=\sum_{y \in Y} p\left(y \mid a_{i}, d_{-i}\right)\left((1-\delta) \pi_{i}\left(a_{i}, y\right)+\delta \kappa_{i}(y \mid d)\right)
$$

Definition 6 Given $\delta \in(0,1)$, for any $W \subset \mathbb{R}^{n}$, a pair $(\mu, \kappa)$, where $\mu$ is a probability distribution on $A, \kappa$ is a function from $A \times Y$ to $W$, is called $D$-admissible with respect to $W$ if

$$
\sum_{d_{-i} \in A_{-i}} \mu(d) h_{i}\left(d_{i}, d, \kappa_{i}\right) \geq \sum_{d_{-i} \in A_{-i}} \mu(d) h_{i}\left(a_{i}, d, \kappa_{i}\right)
$$

for all $d_{i} \in A_{i}, a_{i} \in A_{i}-\left\{d_{i}\right\}$, and $i \in N$.

Definition 7 For $W \subset \mathbb{R}^{n}$, the self-generating operator $B_{D}(W \mid \delta)$ is defined by

$$
\begin{array}{r}
B_{D}(W \mid \delta)=\left\{v \in \mathbb{R}^{n}: v_{i}=\sum_{d \in A} \mu(d) h_{i}\left(d_{i}, d, \kappa_{i}\right), i=1, \ldots, n,\right. \\
\quad \text { for some pair }(\mu, \kappa) D-\text { admissible w.r.t. } W\} .
\end{array}
$$

As usual, the self-generation property holds: if $W$ is a bounded subset of $\mathbb{R}^{n}$ such that $W \subset B_{D}(W \mid \delta)$, then $B_{D}(W \mid \delta) \subset V_{D}(\delta)$. It is also not difficult to show that $V_{D}(\delta)$ is a compact subset of $\mathbb{R}^{n}$. Clearly, disclosing private information at the end of each period introduces public randomization effects in the model. Moreover, augmentation may also contribute to efficiency, which is illustrated in Example 2. 


\section{Two Examples}

In this section, we consider two infinitely repeated games with public monitoring. In Example 1, we show that partial correlation may produce more efficient outcomes than public randomization in an infinitely repeated prisoner's dilemma game with perfect monitoring. The intuition behind the efficiency improvement is the same as in Aumann (1974)'s Example 2.6. However, here it is applied to an infinitely repeated prisoners' dilemma game, in which the stage game has a unique corelated equilibrium. To begin with, we provide some notation for the perfect monitoring case.

The definition of a $C$-admissible pair simplifies to the following: A pair $(\mu, \kappa)$, where $\mu$ is a probability distribution on $A, \kappa$ is a function from $A$ to $W$, is $C$-admissible with respect to $W \subset \mathbb{R}^{n}$ if, for all $a_{i} \in A_{i}, a_{i}^{\prime} \in A_{i}-\left\{a_{i}\right\}$, and $i \in N$,

$$
\begin{aligned}
\sum_{a_{-i} \in A_{-i}} \mu\left(a_{i}, a_{-i}\right)\left((1-\delta) g_{i}\left(a_{i}, a_{-i}\right)+\delta \kappa_{i}\left(a_{i}, a_{-i}\right)\right) & \\
& \geq \sum_{a_{-i} \in A_{-i}} \mu\left(a_{i}, a_{-i}\right)\left((1-\delta) g_{i}\left(a_{i}^{\prime}, a_{-i}\right)+\delta \kappa_{i}\left(a_{i}^{\prime}, a_{-i}\right)\right) .
\end{aligned}
$$

The self-generating operator can be represented as follows:

$$
\begin{array}{r}
B_{C}(W \mid \delta)=\left\{v \in \mathbb{R}^{n}: v=\sum_{a \in A} \mu(a)((1-\delta) g(a)+\delta \kappa(a))\right. \\
\quad \text { for some pair }(\mu, \kappa) C \text {-admissible w.r.t. } W\} .
\end{array}
$$

In Example 1, the set of subgame perfect public randomization equilibrium payoffs is a proper subset of the set of public correlated equilibrium payoffs.

Example 1. The stage game is the following prisoner's dilemma game: 


\section{Player 2}

\begin{tabular}{ccc|c|} 
& Actions & $L$ & \multicolumn{1}{c}{$R$} \\
\cline { 3 - 4 } Player 1 & $U$ & 1,1 & $-b, 2$ \\
\cline { 3 - 4 } & $D$ & $2,-b$ & 0,0 \\
\cline { 3 - 4 } & & &
\end{tabular}

where $b=\frac{2}{5}$. Following Stahl (1991), one can show that, if $\delta \in\left[\frac{b}{2}, \frac{1}{2}\right)$, the set $V_{P}(\delta)$ of subgame perfect public randomization equilibrium payoffs is the triangle $T=\operatorname{co}\{(0,2-b),(2-b, 0),(0,0)\}$. Here $\operatorname{coX}$ denotes the convex hull of $X$.

We first show that $T \subset V_{C}(\delta)$ for all $\delta \in\left[\frac{b}{2}, \frac{1}{2}\right)$.

Claim 1 For all $\delta \in\left[\frac{b}{2}, \frac{1}{2}\right), T \subset V_{C}(\delta)$.

Proof. Given $\delta \in\left[\frac{b}{2}, \frac{1}{2}\right)$, consider the continuation value function $\kappa=(\kappa(U, L), \kappa(U, R)$, $\kappa(D, L), \kappa(D, R)), \kappa(U, L)=\kappa(D, R)=(0,0), \kappa(U, R)=\left(\frac{(1-\delta) b}{\delta}, \frac{2 \delta-b}{\delta}\right), \kappa(D, L)=$ $\left(\frac{2 \delta-b}{\delta}, \frac{(1-\delta) b}{\delta}\right)$. The corresponding game $G^{0}(\delta)=\left(N,\left(A_{i}\right)_{i \in N},\left((1-\delta) g_{i}(a)+\delta \kappa_{i}(a)\right)_{i \in N}\right)$ is as follows:

Player 2

\begin{tabular}{cccc|}
\multicolumn{1}{c}{ Actions } & \multicolumn{1}{c}{$L$} & \multicolumn{1}{c}{$R$} \\
\cline { 3 - 4 } Player 1 & $U$ & $1-\delta, 1-\delta$ & $0,2-b$ \\
\cline { 3 - 4 } & $D$ & $2-b, 0$ & 0,0 \\
\cline { 3 - 4 } & & &
\end{tabular}

Its pure strategy Nash equilibria are $(U, R),(D, L)$, and $(D, R)$, so $\operatorname{co}\{(0,2-b)$, $(0,0),(2-b, 0)\} \subset V_{C}(\delta)$.

We now show that imperfect correlation can produce more efficient outcomes than perfect correlation in the repeated game.

Claim 2 As $\delta$ goes to $\frac{1}{2}$ from below, the distance between $V_{C}(\delta)$ and $(1,1)$ goes to zero. 
Proof. Let $x \in(0,1)$. Suppose that the payoff vector $(x, x)$ can be supported by a pair $(\mu, \kappa)$, where $\kappa(U, L)=(x, x), \kappa(U, R)=(2-b, 0), \kappa(D, L)=(0,2-b)$, $\kappa(D, R)=(0,0)$, and $\mu=\left(\mu_{U L}, \mu_{U R}, \mu_{D L}, \mu_{D R}\right)$ is a probability distribution on $A$ such that $\mu_{U R}=\mu_{D L}>0, \mu_{D R}=0$. The game is symmetric, so we look at it from player 1's point of view. The fact that $(x, x)$ can be supported by $(\mu, \kappa)$ means that

$$
x=(1-\delta)\left(\mu_{U L}+(2-b) \mu_{U R}\right)+\delta\left(\mu_{U L} x+(2-b) \mu_{U R}\right),
$$

and the following two incentive constraints hold:

$$
\begin{gathered}
(1-\delta)\left(\mu_{U L}-\mu_{U R} b\right)+\delta\left(\mu_{U L} x+(2-b) \mu_{U R}\right) \geq 2(1-\delta) \mu_{U L} \\
2(1-\delta) \mu_{U R} \geq(1-\delta) \mu_{U R}+\delta \mu_{U R} x
\end{gathered}
$$

Solving (1) for $x$, we get

$$
x=x\left(b, \delta, \mu_{U R}\right)=\frac{(1-\delta) \mu_{U L}+(2-b) \mu_{U R}}{1-\delta \mu_{U L}}=1-\frac{b \mu_{U R}}{1-\delta+2 \delta \mu_{U R}}
$$

The constraint (2) implies that

$$
x \geq 2(1-\delta)\left(1-\mu_{U R}\right),
$$

and, therefore,

$$
1-\frac{b \mu_{U R}}{1-\delta+2 \delta \mu_{U R}} \geq 2(1-\delta)\left(1-\mu_{U R}\right)
$$

Since

$$
1-\frac{b \mu_{U R}}{\frac{1}{2}+\mu_{U R}}>1-\frac{4}{5} \mu_{U R}>1-\mu_{U R}
$$

we deduce by continuity that for any fixed $\mu_{U R}>0$ there exists $\delta\left(\mu_{U R}\right)<\frac{1}{2}$ such 
that (4) holds for all $\delta \in\left[\delta\left(\mu_{U R}\right), \frac{1}{2}\right)$. The incentive constraint (3) can be rewritten as $x \leq \frac{1-\delta}{\delta}$. This inequality obviously holds for all $\delta<\frac{1}{2}$. It is not difficult to see that $x\left(b, \delta\left(\mu_{U R}\right), \mu_{U R}\right)$ approaches 1 as $\mu_{U R}$ goes to 0.

Adding a public randomization device to an infinitely repeated game is accompanied by redefining the notion of public history, with a public history including not only the history of previously chosen action profiles but also the history of past public messages. As a result, it is easy to find an example where the set $V_{P}(\delta)$ of subgame perfect public randomization equilibrium payoffs strictly contains the set $V_{C}(\delta)$ of public correlated equilibrium payoffs. Moreover, $V_{C}(\delta)$ need not be convex and monotonic in $\delta$ (Prokopovych and Smith 2004), which comes as no suprise since the set of subgame perfect equilibrium payoffs possesses these properties as well.

In repeated games with imperfect public monitoring, the set of perfect public equilibrium payoffs may be bounded away from the Pareto frontier uniformly in the discount rates if the signal space is small (Radner et al. 1986). We will show that augmentation, unlike extensive correlation alone, produces efficiency gains in the game. The fact that players' future payoffs may also depend on the device's recommendations makes the distinguishability problem (the problem of detecting deviators) less severe. At the same time, since the device's recommendations are not binding for players, only realizations of the imperfect public signal may indicate possible deviations.

Example 2. Following Fudenberg et al. (1994), we consider the following repeated partnership game. There are two possible output levels, $y=12$ and $y=0$. If both players work, the probability that $y=12$ is $\frac{2}{3}$, if only one works the probability is $\frac{1}{3}$, and if neither works it is 0 . Each player's utility is half of total output minus the disutility of her effort; work generates a disutility of 3 . Then the players's expected one-period payoffs are given in the following table: 
Player 2

\begin{tabular}{ccc|c|} 
& Actions & $L$ & \multicolumn{1}{c}{$R$} \\
\cline { 3 - 4 } Player 1 & $U$ & 1,1 & $-1,2$ \\
\cline { 3 - 4 } & $D$ & $2,-1$ & 0,0 \\
\cline { 3 - 4 } & & &
\end{tabular}

where the actions $U$ and $L$ correspond to "work" and the actions $R$ and $D$ to "shirk." To simplify notation, we assume that the signal space $Y$ consists of two signals $b$ and $s$, where the symbol $b$ stands for 12 and $s$ for 0 . Then $p(b \mid U, L)=\frac{2}{3}, p(b \mid$ $U, R)=p(b \mid D, L)=\frac{1}{3}$, and $p(b \mid D, R)=0$. Given $\delta \in(0,1)$, we denote by $V(\delta)$ the set of perfect public equilibrium payoffs of this game. A reasoning similar to that provided by Fudenberg and Levine (1994) can be employed to show that the limit set $V_{L}=\lim _{\delta \rightarrow 1} V(\delta)$ is $T=\operatorname{co}\{(0,0),(0,1),(1,0)\}$, the triangle with the extreme points $(0,0),(0,1)$, and $(1,0)$.

First we show that, even with an extensive form correlation device $C$ added, the players' average discounted equilibrium payoffs sum to no more than 1 . The proof of this statement is similar to that provided by Fudenberg et al. (1994) for perfect public equilibrium payoffs.

Claim 3 For all $\delta \in(0,1), V_{C}(\delta) \subset \operatorname{co}\{(0,0),(0,1),(1,0)\}$.

Proof. We first show that if $v=\left(v_{1}, v_{2}\right) \in V_{C}(\delta)$, then $v_{1}+v_{2} \leq 1$. Let $\gamma(\delta)=$ $\max \left\{v_{1}+v_{2} \mid\left(v_{1}, v_{2}\right) \in V_{C}(\delta)\right\}$. By contradiction, assume that $\gamma(\delta)>1$ for some $\delta \in(0,1)$. Let $v=\left(v_{1}, v_{2}\right) \in V_{C}(\delta)$ be such that $v_{1}+v_{2}=\gamma(\delta)$. It can be decomposed in a $C$-admissible pair $\mu \in \triangle(A)$ and $\kappa: Y \mapsto V_{C}(\delta)$. Then

$$
\begin{aligned}
v_{1}= & \mu_{U L}\left((1-\delta) 1+\delta\left(\frac{2}{3} \kappa_{1}(b)+\frac{1}{3} \kappa_{1}(s)\right)\right) \\
& +\mu_{U R}\left((1-\delta)(-1)+\delta\left(\frac{1}{3} \kappa_{1}(b)+\frac{2}{3} \kappa_{1}(s)\right)\right) \\
& +\mu_{D L}\left((1-\delta) 2+\delta\left(\frac{1}{3} \kappa_{1}(b)+\frac{2}{3} \kappa_{1}(s)\right)\right)+\delta \mu_{D R} \kappa_{1}(s)
\end{aligned}
$$


and

$$
\left(\mu_{U L}+\mu_{U R}\right)\left((1-\delta)(-1)+\delta\left(\frac{1}{3} \kappa_{1}(b)-\frac{1}{3} \kappa_{1}(s)\right)\right) \geq 0
$$

Since $\gamma(\delta)>1, \mu$ puts positive probability on the action profile $(U, L)$. Player 1's second incentive constraint is omitted because it has no bearing on the below reasoning. It follows from (6) that

$$
\kappa_{1}(b)-\frac{3(1-\delta)}{\delta} \geq \kappa_{1}(s)
$$

Substituting for $\kappa_{1}(s)$ in $(5)$, we have

$$
v_{1} \leq \delta \kappa_{1}(b)
$$

By symmetry,

$$
v_{2} \leq \delta \kappa_{2}(b)
$$

This leads to the conclusion that $v_{1}+v_{2} \leq \delta \gamma(\delta)$, which contradicts the initial assumption.

Adding an extensive form correlation device to the repeated game does not make available public information richer. Moreover, the signal space is too poor for the correlation to have any efficiency-improving effect. At the same time, adding an augmented correlation device to the game improves efficiency because its payoff-irrelevant recommendations enrich public histories, and, as a result, future payoffs may depend on both the history of realized public signals and the history of the device's payoffirrelevant recommendations.

Claim 4 For all $\delta \in\left[\frac{3}{4}, 1\right)$, the set $T=\operatorname{co}\{(0,0),(0,1),(1,0)\}$ is strictly contained in $V_{D}(\delta)$

Proof. We first show that $T \subset V_{D}(\delta)$ for $\delta \in\left[\frac{3}{4}, 1\right)$. Let $\delta \in\left[\frac{3}{4}, 1\right)$ and the continuation 
value function $\kappa$ be such that $\kappa(b \mid U, R)=\left(\frac{3(1-\delta)}{\delta}, 1-\frac{3(1-\delta)}{\delta}\right), \kappa(s \mid U, R)=(0,1)$, $\kappa(b \mid D, L)=\left(1-\frac{3(1-\delta)}{\delta}, \frac{3(1-\delta)}{\delta}\right), \kappa(s \mid D, L)=(1,0), \kappa(b \mid D, R)=\kappa(s \mid D, R)=$ $(0,0)$, and $\kappa(b \mid U, L)=\kappa(s \mid U, L)=(0,0)$.

Player 1's incentive constraints are the following:

$$
\begin{aligned}
\mu_{U L}((1-\delta)(-1)+ & \left.\delta\left(\frac{1}{3} \kappa_{1}(b \mid U, L)-\frac{1}{3} \kappa_{1}(s \mid U, L)\right)\right) \\
& +\mu_{U R}\left((1-\delta)(-1)+\delta\left(\frac{1}{3} \kappa_{1}(b \mid U, R)-\frac{1}{3} \kappa_{1}(s \mid U, R)\right)\right) \geq 0
\end{aligned}
$$

and

$$
\begin{aligned}
\mu_{D L}((1-\delta) 1+\delta(- & \left.\left.\frac{1}{3} \kappa_{1}(b \mid D, L)+\frac{1}{3} \kappa_{1}(s \mid D, L)\right)\right) \\
& +\mu_{D R}\left((1-\delta) 1+\delta\left(-\frac{1}{3} \kappa_{1}(b \mid D, R)+\frac{1}{3} \kappa_{1}(s \mid D, R)\right)\right) \geq 0
\end{aligned}
$$

Since

$$
\begin{aligned}
\left(h_{1}\left(U,(U, L), \kappa_{1}\right), h_{2}\left(L,(U, L), \kappa_{2}\right)\right) & =(1-\delta, 1-\delta), \\
\left(h_{1}\left(U,(U, R), \kappa_{1}\right), h_{2}\left(R,(U, R), \kappa_{2}\right)\right) & =(0,1), \\
\left(h_{1}\left(D,(D, L), \kappa_{1}\right), h_{2}\left(L,(D, L), \kappa_{2}\right)\right) & =(1,0), \\
\left(h_{1}\left(D,(D, R), \kappa_{1}\right), h_{2}\left(R,(D, R), \kappa_{2}\right)\right) & =(0,0),
\end{aligned}
$$

the containment $T \subset V_{D}(\delta)$ holds if $(\mu, \kappa)$ is a $D$-admissible pair for every $\mu=$ $\left(\mu_{U L}, \mu_{U R}, \mu_{D L}, \mu_{D R}\right) \in \triangle(A)$ such that $\mu_{U L}=0$, which follows straightforwardly from (8) and (9). Because of symmetry, there is no need to check player 2's incentive constraints.

Now we show that there exists $(x, x) \in V_{D}(\delta)-T$ that can be supported by a public augmented equilibrium. Let $x \in(0,1)$ and $\kappa$ be such that $\kappa(b \mid U, L)=\kappa(s \mid U, L)=$ 
$(x, x), \kappa(b \mid U, R)=(1,0), \kappa(s \mid U, R)=\left(1-\frac{6(1-\delta)}{\delta}, \frac{6(1-\delta)}{\delta}\right), \kappa(b \mid D, L)=(0,1)$, $\kappa(s \mid D, L)=\left(\frac{6(1-\delta)}{\delta}, 1-\frac{6(1-\delta)}{\delta}\right), \kappa(b \mid D, R)=\kappa(s \mid D, R)=(0,0)$. Because of symmetry, we look only at player 1's payoff. It is easy to check that the incentive constraints (8) and (9) hold for $\mu=\left(\frac{1}{3}, \frac{1}{3}, \frac{1}{3}, 0\right)$. So, if $x$ is decomposable with respect to $\mu=\left(\frac{1}{3}, \frac{1}{3}, \frac{1}{3}, 0\right)$ and $\kappa$, then

$$
\left.x=\frac{1}{3}((1-\delta) 1+\delta x)+\frac{1}{3}\left((1-\delta)(-1)+\delta\left(1-\frac{4(1-\delta)}{\delta}\right)\right)\right)+\frac{1}{3}((1-\delta) 2+4(1-\delta)) .
$$

After simplifying, we have

$$
x=\frac{2-\delta}{3-\delta}
$$

It is clear that $2 x>1$ for any $\delta \in\left[\frac{3}{4}, 1\right)$.

The key to the efficiency improvement is the following. If the profile of privately recommended actions is $(U, L)$, then player 1's deviation to $D$ or player 2's deviation to $R$ would go unpunished. However, if the profile of privately recommended actions is $(U, R)$, a severe punishment would follow player 1's deviation to $D$. Moreover, after getting the recommendation "play $U$," player 1 does not know with certainty the action recommended to player 2, which keeps player 1 from deviating to $D$. Therefore, augmentation helps alleviate the efficiency-reducing condition (7). This condition is also to be blamed for the fact that the maximum value a player can get in a strongly symmetric perfect public equilibrium of the game is much smaller than one half (Abreu et al. 1991). Even switching to private strategies does not result in payoffs vectors lying outside $T$ (Kandori and Obara 2006a). Though Kandori and Obara's idea to check the partner's behavior by randomization is present here in its extreme form: The bad public signal does not cause any punishment if the profile of privately recommended actions is $(U, L)$. Thus, in the repeated partnership game, 
disclosing private payoff-irrelevant messages at the end of each period makes efficiency improvements possible.

Acknowledgements I thank Roy Gardner, Lones Smith, and Ennio Stacchetti for helpful discussions and valuable suggestions.

\section{References}

Abreu D, Milgrom P, Pearce D (1991) Information and timing in repeated partnerships. Econometrica 59:1713-1733

Abreu D, Pearce D, Stacchetti E (1990). Toward a theory of discounted repeated games with imperfect monitoring. Econometrica 58:1041-1063

Aumann R (1974) Subjectivity and correlation in randomized strategies. J Math Econ 1:67-96

Forges F (1986) An Approach to Communication Equilibria. Econometrica 54:137585

Fudenberg D, Levine D (1994) Efficiency and observability with long-run and short-run players. J Econ Theory 62:103-135

Fudenberg D, Levine D, Maskin E (1994) The folk theorem with imperfect public information. Econometrica 62:997-1040

Kandori M (2003) Randomization, communication, and efficiency in repeated games with imperfect public monitoring. Econometrica 71:345-353

Kandori M, Obara I (2006a) Efficiency in repeated games revisited: the role of private strategies. Econometrica 74:499-519

Kandori M, Obara I (2006b) Less is more: an observability paradox in repeared games. Int J Game Theory 34:475-493

Lehrer E (1992) Correlated equilibria in two-player repeated games with nonob- 
servable actions. Math Oper Res 17:175-199

Myerson R (1986) Multistage games with communication. Econometrica 54:32358

Myerson R (1991) Game theory: Analysis of conflict. Harvard University Press

Prokopovych P, Smith L (2004) Subgame perfect correlated equilibria in repeated games. Econometric society North American summer meetings.

No. 287, http://repec.org/esNASM04/up.19092.1075409728.pdf

Radner R, Myerson R, Maskin E (1986) An example of a repeated partnership game with discounting and with uniformly inefficient equilibria. Rev Econ Stud 53:59-69

Stahl II D (1991) The graph of prisoners' dilemma supergame payoffs as a function of the discount factor. Games Econ Behav 3:368-384

Tomala T (2005) On subgame-perfect communication equilibria in repeated games with imperfect monitoring. mimeo 